longs, lestace-rougraitre, avec les élytres et le devant de la tete do testacé pàle; tète brierement maculée près des y'eux et prothorax st le disque orné de deux grandes bandes noires; élytres ornés chace de deux groses macules noires, l'une humérale, l'autre plus gran avant le sonmet, ni l'une ni l'autre n'atteignant la suture; antenn moins la base, et pattcs sauf d'ordinaire l'extrème base des cuisse noires. Prothorax presque lisse, ainsi que la tète, assez court, sii arrondi sur les colés; élytres assez linement ponctués, munis d’ü carene latérale anterieure, bien plus larges que te prothorax à la base (ilirgis postérieurement. - Long. 33 mill. euriron.

Brisil : Bahia (coll. Pic).

Voisin de l'1. julayensis Pic, mats de coloration très différente, se rapprochant darantage le colle de l.1. Urasiliensis Pic; d'aillei bien cartactirisi par les deux bandes noires paralleles du prothoras

\title{
Diagnoses de nouveaux genres et de nouvelles espèces de Scélionides [Him.] des îles Séchelles
}

\section{par J.-J. KIFFHER.}

Paranteris. n. g. - leux pubrecents, mondibules tridenteses falges maxilliares de 3 irtiches. les libiand de 2 . Antennes de 12 ar ticles, celles de lit remelle aree me massur de 6 articles. Nervure marwimale ponclifurme. postmarrinale nulle on pas plus longue que b stigmatione. Abdumen spatulifurme. Ler lergite de la femelle allonges arec une próminence.

T!pe: P. nigriclava, n. sp. - P'ustmalrginale nulle, articlés $3 \cdot 1 /$ des inlennes du mile trois it quatre fois aussi longe que gros, antennes de lit femelle frunes, massue nuire, sillons parapsidaux distincts.

P. flaviclava, ll. sp. - Dilliere du lype par la massue jaune,

P. nigraticeps, n. sp. - Dillire du l! pe par les sillons parapss doux indistincts ou nuls; lous les tergites striés.

P. striatigena, n. sp. - Dillere du précédent par les tergites 40 linement ponctuis.

P. nitidiceps, n. sp. - Dilfëre du lype par lit prísence d'une post. marginale. 
P. densiclava, n. sp. - Diffère du précédent par la massue anfaire non distinctement articulée.

Lamproteleia, n. g. - leux glabres. Antennes du précédent. ginale plus longue que la sligmatique, postmarginale nulle. Abdofusiforme, $1^{\text {er }}$ tergite de la femelle allongé, avec une proémiace.

Type: L. fasciatipennis, n. sp. - Sillons parapsidaux percur-

L. pulchripennis, n. sp. - Sillons parapsidaux nuls.

veuroteleia, $n . \stackrel{\text { ir }}{s}$ - Ailes a nervation complete, marginale plus ourte que la stigrmatique. Yeux glabres. Yétanotum avec deux dents. Homen fusiforme. $1^{\text {er }}$ territe avec une petite corne.

fype: $\mathbf{N}$. rufa, n. sp. - Antennes de la femelle avec une massue 6 articles.

1. heterocera, 17. sp. - Antennes de la temelle sans massue.

Enneascelio, n. ir. - Comme Scelio mais prothorax autrement miormé el massue antennaire de la femelle de 9 artictes.

Type : E. exaratus, 11. sp. - Voir, paltes jaunatres en partic.

Oreiscelio. n. g. - Diltere de Scelio par le scutellum afui est bi. lenté.

Type : $O$. sechellensis, n. sp. - Yoir, articles antennaires $\nexists-6$ et gattes jaunes.

Psiloteleia atra, n. sp. - Yoir. pattes jaunes, antennes hruntes. massue de la femelle noire. scape du mile jaune.

Badronotus festivus, n. sp. - Rom, antennes. tible rt muitie pstérieure de laaldomen noires.

Eadronotus saxatilis. n. in. - Vir, scape, genoux, libias "l arses testacés, articles 2 - $k$ des antemnes de la lemelle bruns.

Yacroteleia mahensis, n. sp. - Roux, massue antennaire "t Homen noirs, 4e article des antennes de la femelle deux fois anssi bag que gros.

Macroteleia versicolor, n. sp. - o nuir, pattes et deux premiers Wicles antennatres jaunes: f rousse, variese de noir, $4^{e}$ article des atemes à peine plus long yue gros. terorites a-' deux fois aussi longs pe larges.

Kacroteleia flavigena, n. sp. - Kuir; bas de la lite et paltes anos, qu $^{\mathrm{a}}$ article antennaire i peine phus long que gros, tergites $2-4$ i ribe plus longs que larges. 
Baryconus calopterus, n. sp. - Diffère des congénères ailes qui sont enfumées dans la moitiè distale et ont une mince transversale dans la moitié proximale.

Paratrimorus atriceps, n. sp. - Roux brun, tête et m noires, pattes et deux premiers articles antennaires jaunes.

Acolus zonatus, n. sp. - Noir, pattes et $1^{\text {er }}$ tergite jaunese mate et chagrinée.

Acolus lucidiceps, n. sp. - Diffère du précédent par la tetch et brillante.

Acoloides unicolor, n. sp. - Entierement jaune pile, fer for n pétiole.

Odontacolus, n. g. - Differe de Ceratobaeus par le mêta: bidenté et la presence d'une postmarginale.

Type : . O. longiceps, n. sp. - Jaune, tite noire, articles antenin 2- 6 et thorax bruns.

Ceratobaeus insularis, 0 . sp. - Noir, antennes, 1 er tergit pattes sauf les hanches et les fímurs, jaunes.

Parabaeus, n. g. - Diffère de Bueus par la massue, antenz composée de 4 articles connés, le pronotum et la forme de l'abdon

Type: P. ruficornis, n. sp. - Yioir. antennes et pattes rouges

Baeus curvatus, n. sp. - Coir, antennes et pattes jaunes, oedta nuls.

Telenomus sechellensis, n. sp. - Noir, scape el pattes les hanclies jaune vitellin. Tite lisse et brillante, yeux glabres, ablo. men igal au thorax $(q)$ ou plus court $\left(O^{*}\right)$, ze tergite strié en arant

Telenomus myrmidon, n. sp. - Yoir. pattes sauf les hanches jaune brunditre. Tête lisse et brillante, yeux velus, abdomen pluscoun que le thorax, we tergite non stric.

Telenomus mahensis, n. sp. - Yoir, pattes testacees, scape la femelle testacé. Tète mate et chagrinée, yeux glabres, abdomes égal au reste du corps, 2 e tergite strie a la base. 